NASA Technical Memorandum 100201

AIAA-87-2722

\title{
Control of Shear Flows by Artificial Excitation
}

\author{
(NASA-TM-100201) CONTROL OF SHEAR FLOUS BY \\ ARTIFICIAL EXCITATION (NASA) 12 p Avail: \\ NTIS HC AO2/MF AO 1 \\ CSCL OIA

E.J. Rice and K.B.M.Q. Zaman

Lewis Research Center

Cleveland, Ohio

Prepared for the

11th Aeroacoustics Conference

sponsored by the American Institute of Aeronautics and Astronautics

Sunnyvale, California, October 19-21, 1987 


\section{Abstract}

Investigations involving artificial excitation of various shear flows are reviewed. Potential applications of excitation in flow control, F e.g., in enhancing mixing, and in delaying transition and separation are discussed. An account is L' given on the current activities at NASA Lewis Research Center in this regard.

\section{Introduction}

In the last 50 years or so, artificial excitation has been widely used as an experimental tool to advance our understanding of shear flow dynamics. The concepts of flow instability leading to transition, and of the large scale coherent structures in turbulent shear flows have prompted numerous experiments involving excitation. The purpose of the excitation has been to introduce a disturbance to be followed in space or time for the instability study, or to organize the coherent structures so that they could be "educted" with relative ease from the background randomnesses of a turbulent flow. Experience from these studies has also led to the possibility of utilizing excitation to achieve control of certain types of flow phenomena, e.g., boundary layer transition and separation, and shear layer mixing. In this paper we discuss the general scope of excitation as a tool of fluidmechanics research, with emphasis on experimental studies and on aspects of flow control. A review is given first, followed by a discussion on current and future efforts at NASA Lewis in this connection.

\section{Reriew}

The principal applications of artificial excitation have been in the studies of: (a) flow instability, (b) large scale coherent structures, and $(c)$ control of various flows. These interrelated aspects of fluidmechanical research are further categorized in Table 1 and discussed below.

\subsection{Flow Instability}

Stability analysis based on the small amplitude perturbation of flows is regarded today as a powerful tool providing an understanding of the events leading to transition. In the beginning, however, there was skepticism as, "in the late thirties the prevailing view was....that stability theory had little or no connection with boundary layer transition,". I It was Schubauer and Skramstad's ${ }^{2}$ landmark experiment, revealing the growing two-dimensional waves prior to transition in a flat plate boundary layer, that firmly supported the earlier analytical predictions of Tollmien and Schlichting. Klebanoff et al. ${ }^{3}$ in a later experiment observed a distinct secondary instability in the growing "Tollmien-Schlichting" waves. This was later analyzed by Craik, 4 and further by Herbert; 5 Kachanov and Levchenko, 6 among others, reported further experimentation on the subject. The basic concept in Craik's analysis, viz. the interaction of two oblique waves with the fundamental T-S wave, has been put to use by Corke and Mangano 7 in experiments studying the secondary instability in a controlled manner. Arrays of heating strips with phased signals were used to introduce two oblique waves; the phasing determined the included angle which in turn controlled the spanwise wavenumber.

By far the most extensive analysis of the stability of a free shear layer has been carried out by Michalke. 8 Freymuth's 9 experiment in an acoustically excited shear layer confirmed Michalke's predictions of the growth rates associated with. different Strouhal number components. Michalke10 also analyzed the axisymmetric mixing layer, taking into account the ratio of the jet diameter (D) to the shear layer momentum thickness $(\theta)$ as a parameter, and found general agreement with the results of Crow and Champagne's 11 experiment. Bechert and Pfizenmaleris in a subsequent experiment confirmed Michalke's prediction of phase velocities greater than the jet velocity occurring at low Strouhal number and for large $D / \theta$. The instability of helical mode disturbances in axisymmetric jets has been studied, among others, by Ahuja et al. 13 and Strange and Crighton. 14 ' Cohen and Wygnanski 15 recently considered the modal distributions of coherent structures both analytically and experimentally; this will be discussed in some detail in section 3 .

Artificial excitation has also been used to study the instability of many other free flows, e.g. that of slit jets, 16 of wakes, 17 of elliptic jets, 18,19 and of hot jets. 20

\subsection{Large Scale Coherent Structure Study}

The concept of large-scale coherent structures in turbulent shear flows 21 has provided a tremendous impetus in turbulence research. The notion of orderliness in these structures, responsible for most of the transport, noise production, etc., produced the enthusiasm and expectation that a knowledge of their characteristics may lead to viable turbulence models and theories. Thus, there has been a number of experiments conducted to measure the characteristics of these structures. Artificial excitation in these studies has been used to a somewhat different end. Because of the randomness in the characteristics of successive structures one needs to perform elaborate conditional sampling measurements in order to "educt" the average properties. Appropriate excitation, however, organizes the structures and raises them "to high amplitude above the random background" and thus renders them easier to study. 11

There are situations where the "preferred structures" in the natural flow are made periodic by appropriate excitation, in which case the conditional sampling, employed for the eduction, reduces to the relatively simple and less ambiguous phase averaging. This was the basic idea utilized in many experiments, e.g. 22-24 Controlled excitation 
made it possible to study the interactions, among the coherent structures as well as between the coherent and the incoherent fields, 22 in such detail as would be impossible otherwise. Such knowledge gathered so far on the coherent structure dynamics has yet to be included profitably in any turbulence model. However, certain numerical analyses, 25 treating the coherent structures as a set of harmonics of instability waves, have done quite well in predicting the gross features of the flow.

\subsection{Flow Control}

The third main application of artificial excitation has been in attempts to control or suitably modify various flows with technological benefits in mind. The aim here is to achieve desired changes in the flows, e.g., enhanced mixing or reduced separation, in order to gain higher efficiencies in fluidmechanical devices. This is the general focus of the activities of the group at NASA Lewis to which the authors belong. The approach taken here can often be somewhat less rigorous, sometimes involving trial and error, which is well justified by the complexities of the flows involved. However, successful research in this area must draw on the experience from the fundamentals, going hand in hand with the areas covered in sections 2.1 and 2.2 .

The research activity in flow control can be further subdivided in categories as shown in Tabie 1. We concern ourselves only with methods involving controllable, artificial excitation. There are other approaches including many promising "passive" methods; an excellent review of research on turbulent boundary layer drag reduction has been given by Bushne 11.26 The possibilities of boundary layer transition control have been discussed by Reshotko. 27 stone and Mckinzie 28 discussed the potential of acoustic excitation in flow control.

2.3.1 Enhanced mixing. Past experimental studies indicate that excitation can increase turbulence and enhance mixing in shear flows resulting in increased spread rates, reduction in jet plume temperature, etc. $11,13,29$ Jet plume temperature reduction is sought to cut down on the infra-red signature, to reduce wing surface heating in overor under-the-wing engine configurations, as well as in Vertical Takeoff and Landing aircraft to avoid ground erosion, hot gas ingestion, etc. 28 other potential applications are in chemical process industries and in the improvement of combustion and convective heat transfer efficiencies. Current research on the effect of acoustic excitation on jet mixing is further discussed in section 3 .

\subsubsection{Turbulence suppression. Certain high} frequency excitation of jets and shear layers can reduce turbulence levels. A comprehensive documentation of the phenomenon occurring in round and slit jets, as well as single mixing layers, was provided in Ref. 30 . It turns out that excitation at frequencies about 40 percent higher than the natural initial roll up frequency of the shear layer produces the effect. The effect is very pronounced in flows with initially laminar or transitional boundary layers but diminishes as the initial boundary layer approaches a fully turbulent state. 31
2.3.3 Boundary layer transition control. The possibility of delaying transition in boundary layers has been explored in several experiments. T-S waves introduced by one vibrating ribbon have been successfully cancelled by anti-phased waves introduced by a second, downstream ribbon. 32-33 However, cancelling natural T-S waves, and thus significantly delaying the transition otherwise occurring in the natural boundary layer, has not been successfully demonstrated yet.

On the other hand, enhancing transition in boundary layers or "transition fixing" by artificial excitation seems quite feasible. 34 This has the potential application in separation control as discussed in the following subsection.

2.3.4 Separation control. The authors believe that a large potential exists for performance improvement in various fluid mechanical devices through control of separation. "Passive" tripping devices are widely used to force transition of laminar boundary layers or energize turbulent boundary layers so that separation is delayed. This significantly reduces profile drag (an often cited application is the "dimpled golf ball"), while increasing the lift of airfoils. However, such passive separation control usually involves trial and error. The tripping device that may work in one flow situation may not only be ineffective in another but also represent a drag penalty at higher Reynolds number. 35 "Active" separation control (i.e., with the ability to change parameters in the controlling device according to need), through artificial excitation, appears much more promising in this regard. In the following, the items under this subheading in Table 1 are further discussed.

Flow over airfoils/blades: The effect of acoustic excitation in improving lift and delaying stall for two-dimensional airfoils has been studied, among others, by Ahuja and Burrin 36 and Zaman et al. 37 In the experiment of Ref. 36 , extensive data were obtained in post-stalling conditions covering Reynolds numbers up to a million. It was shown that excitation not only improved the stall margin but also reduced the severity of stall, i.e., the lift coefficient was increased even in the post-stalling angles of attack. It was inferred that the improvement achieved with excitation diminished with increasing Reynolds number, however, the excitation frequency and amplitude required to control separation on airfoils ought to increase with increasing Re. The effectiveness of higher frequencies and levels of excitation at high Re remains to be investigated.

In the experiment of Ref. 37 , carried out at low Re, the effect of wind tunnel resonance on the results has been addressed. It was shown that tunnel resonances inducing large cross-stream velocity perturbations, rather than ones inducing large pressure perturbations, near the airfoil were most effective in separation control. Thus, excitation methods specifically inducing cross-stream velocity perturbation appear more promising. In an on-going experiment at NASA Langley, a vibrating wire located in front of the leading edge of an airfoil (inducing the cross-stream velocity perturbation), at low $\mathrm{Re}$, has produced more pronounced separation control (A. Bar-Sever, private communication).

Turbomachinery. The flows in turbomachinery are very complex but the performance limitations 
also arise from flow separation over the blades. These manifest themselves in terms of blade row "stall margin" and also as more complex phenomena such as rotating stall, dynamic stall, etc. It is conceivable that active excitation of the flows over individual blades or even global excitation of the flow may improve turbomachinery performance. Some of these possibilities have been discussed in Ref. 38 which also cites an experiment in which improvement in the performance of a compressor was achieved by using only a loudspeaker.

Dynamic effects on stall. Stalling together with unsteady flow effects is referred to as dynamic stall. The combined translating and rotating motion of a helicopter blade is most commonly cited as an example of dynamic stall; the effective pitch of the blade varies during a revolution and thus may subject the blade to cyclic stalling and unstalling. Dynamic stall can result in undesirable structural vibrations resulting in "stall flutter" of propellers, compressor blades, etc. (McCroskey ${ }^{39}$ provides a comprehensive review). The phenomenon is usually simulated in a wind tunne? by periodically oscillating an airfoil in pitch. 40 The physical process in dynamic stall involves a "vortex-like" disturbance originating from the leading edge region, possibly due to the bursting of a leading edge bubble, and passing over the upper surface of the airfoil during a cycle of the unsteadiness 39 . Again, since the phenomenon involves a leading edge bubble and separation, it is conceivable that some amount of control could be achieved by artificial excitation. An experiment involving an oscillating airfoil together with acoustic excitation is in its preliminary stages at NASA Lewis.

Diffuser Separation. Separation control in diffusers, inlets and nozzles and transition ducts is an important area of active flow control research. Viets et al.41 have shown the influence of unsteady flow perturbations on the flow reattachment of separated two-dimensional diffusers. They produced vorticity oscillations (with spiral shaped rotors mounted in the walls) which flowed into the separation region of the diffuser. Reattachment was demonstrated using both flow visualization and wall measurements which showed a diffuser performance improvement with the reattached flow. A vital element of active diffuser separation control work is to find excitation devices that have sufficiently low aerodynamic losses to provide practical solutions to the problem. Simpson 42 provides a review of separation and reattachment phenomena in diffusers, over blunt bodies and backward facing steps, etc.

\subsection{Methods of Excitation:}

In boundary layer stability and control experiments, T-S waves have been typically introduced by vibrating ribbons. $2,3,6,33$ Flush mounted heating strips, introducing periodic heat flux, have also been used. $7,32,34$ Heating strips, however, are much more effective in water than in air. It was shown in 32 that for a given amplitude of temperature fluctuation, the amplitude of crossstream velocity fluctuation induced in air is about twenty times less than that in water. Thus, heating strips do not seem to be promising as large amplitude excitation device in air. 43 However, for purposes of triggerring transition in many situations they seem to be adequate. 34 Acoustic excitation has also been used to introduce $T-S$ waves, e.g. 44 The "receptivity" of the boundary layer to such excitation has been analyzed by Goldstein, e.g. 45

In free shear flows, acoustic excitation has been used very extensively. Acoustic drivers placed upstream in the plenum chamber have been used to excite jets, usually utilizing the settling chamber cavity resonances. $11,12,29,31$ The excitation is in the form of a periodic fluctuation of the flow which is uniform at the exit plane. Besides this "internal" excitation, "external" excitation of only the annular shear layer near the jet exit has also been employed by circumferential arrays of drivers. 15,20,46 A circumferential array of acoustic drivers was also used to excite helical mode instabilities in round jets. 13-15 Other methods of excitation in both free and wall bounded flows include vibrating wires, 47 flaps, 24 sparks, ${ }^{48}$ corona discharge, 26 etc.

\section{Efforts at NASA Lewis}

The NASA Lewis effort on "control of shear flows by artificial excitation," the description of which is the main emphasis of this paper, is carried out in the Internal Fluid Mechanics Division. The work is an outgrowth of the jet noise research program. The effort is carried on by seven researchers, six performing mainly experimental work and one performing numerical experiments. A significant contract and grant program has been in place to supplement the in-house program. Each effort will be briefly summarized below. The main emphasis of the program is flow control but the efforts on fundamental understanding of the physics, required to successfully control the flow, should provide many side benefits in better understanding unsteady aerodynamics and turbulence.

\subsection{In-house}

The several elements of the in-house program are summarized below.

3.1.1 Initial turbulence effect on the excitability of a jet. Natural jet evolution was found to remain unaffected for different initial turbulence levels varied (using grids) over the range 0.15 to 5 percent. Tonal excitation, with velocity perturbation amplitude of as little as 0.25 percent of the jet velocity, measurably altered all jets; however, for a fixed amplitude of excitation, the excitability diminished systematically with increasing initial turbulence. Jets with initially laminar boundary layers decayed faster naturally; consequently, they were less susceptible to tonal excitation. Further details are discussed in Ref. 29.

3.1.2 Excitation of swirling jets. The effect of acoustic excitation on an axisymmetric, cold jet with and without swirl has been studied experimentally.49 Plane wave, single frequency excitation was imparted to the jet "internally" by acoustic drivers placed upstream in the plenum chamber. Comparative velocity fluctuation measurements were performed on the jet axis, using a hot-wire, for a case with a swirl number of 0.35 and a case without swirl but with the same initial, axial mass flux. It has been found that the swirling jet is excitable; however, the instability wave grows 
about 50 percent less in peak rms amplitude, and saturates farther upstream compared to the corresponding waves in the jet without swirl. Measurements made 2.5 diameters downstream of the nozzle exit with varying frequency but fixed amplitude of the excitation, show that in both cases the instability wave receives maximum amplification at a Strouhal number of about 0.4 , the latter being based on the mass averaged axial velocity and the jet diameter.

3.1.3 Experiments on airfoils. The effect of acoustic excitation in removing laminar separation, at small angles of attack and at low Re, has been studied experimentally. Experiments were conducted to determine the frequency dependence of this process; preliminary results from four different airfoils show that excitation at about fu/ $/ 0^{2}=10^{-5}$ is most effective; here, $f$ is the excitation frequency, $v$ the kinematic viscosity and $U_{0}$ the free-stream velocity. Detailed hot-wire surveys indicate that the excitation effect takes place in the separated shear layer and not in the upstream boundary layer.

An unusually low frequency unsteady flow in the wake of an airfoil, first reported in Ref. 37 and seemingly associated with stall flutter, is under investigation. The unsteady oscillation occurs naturally in the wake of a rigidly held airfoll at a frequency about an order of magnitude lower than the usual "bluff body shedding" frequency. It appears akin to low frequency unsteadiness in transitory stall in diffusers. 42 The oscillation originates from near the leading edge and is felt strongly on the upper surface of the airfoil. It exerts strong unsteady forces on the airfoil; if the airfoil is loosened from the rigid mount it starts fluttering. The phenomenon has been found to occur in flows with relatively high turbulence intensity but not in cleaner flows. It is also found to be controllable by high frequency acoustic excitation. Detalled experimentation is under way, and some results will be discussed in the oral presentation.

3.1.4 High amplitude excitation device. Excitation devices may be classified into two categories: those used for research purposes and those which can be used to excite and control a flow of practical interest. In the first category, ordinary acoustic drivers, oscillating flaps, etc. might prove sufficient for most purposes especially in low Mach number flows. However, as Mach number is increased and background noise and turbulence level increase, there is a struggle to attain sufficient excitation amplitude to influence the flow. Ahuja et al 20 have gone to eight externally mounted drivers located right at the nozzle exit. They are able to attain a sound pressure level at the nozzle exit centerline of $147 \mathrm{~dB}$ over a reasonable frequency range. The external driver arrangement is also advantageous in that arbitrary modes can be driven in the shear layer whereas with the "internal excitation" only axisymmetric modes, in the useful frequency range, can be driven due to acoustic cut-off in the nozzle. In order to increase the excitation capability, in-house tests have been conducted using an electro-pneumatic driver with the removal of the steady flow driving the device. The steady flow (up to $0.551 \mathrm{bm} / \mathrm{sec}$ per driver) is undesirable since it would disturb the flow under study. The device successfully removed the steady flow but allowed the oscillating waves to pass. The results will be reported in the future.

In the second category of devices to excite practical flows, certain properties are desirable. The device should preferably have a minimal power requirement, no moving parts, and should be compact and adjustable in frequency and amplitude over reasonable ranges. Pneumatic or fluidic oscillators are a class of devices that may satisfy these requirements. The simple slot resonator is an example in this class which has been studied extensively. 50-52 An example of the use of the slot resonator would be to locate it just inside the nozzle lip of a jet to produce large vortical fluctuations. The design of such a device must consider several factors. First, the separating shear layer at the upstream edge of the slot has its own instability frequency range. This must be properly matched to the cavity dimensions to produce high amplitude response in the cavity. Secondly, the entire device must then be matched to the jet "preferred frequencies" scaling on the shear layer thickness and the jet diameter. Finally, the slot excitation could be stimulated by some "tickler" device to achieve some control on the frequency and amplitude as well as mode shape. In-house experiments on such a device are currently being conducted. The flip-flop jet nozzle studied by Viets 53 and the whistler nozzle studied by Hasan and Hussain 54 are other exmples of fluidic oscillators used to excite shear layers.

3.1.5 Shear layer linear stability calculations. Calculations of the growth of disturbances in the jet shear layer using the theory of Michalke 55 have been made. 56,57 Measured velocity profiles of co-annular flows, having a hot primary jet, have been used. The flows represent realistic turbofan engine exhaust conditions and the profiles are obtained from actual two-stream experiments. The calculations have provided useful relative instability characteristics for different conditions and configurations. The frequency range of instability is observed to broaden when the outer jet is added to the primary jet. For both the axisymmetric and first circumferential mode, a higher secondary (outer) to primary velocity ratio $(0.68$ as opposed to 0.3$)$ greatiy increases the mode instability while heating the secondary jet reduces the instability.

3.1.6 Diffuser separation control. Controlling separation in propulsion system inlets, diffusers and transition ducts is very important. Active control of these flows using excitation will be a major part of the NASA Lewis research program. A first step in this direction involved the study of separation control of the flow over a twodimensional diffuser ramp located on the floor of the NASA Lewis 20 - by $30-i n$. Wind Tunnel. The ramp had a fairly rapid rise (consequently, flow area contraction) followed by a $20^{\circ}$ downstream diffusion. When the boundary layer was laminar, the flow separated at the beginning of the diffusing section. A small oscillating airfoil was located on the surface at the separation point. A small magnitude oscillation of this airfoil caused complete reattachment of the flow with pressure recovery occurring all along the downstream ramp. However, when the tunne! velocity was increased and the boundary layer became turbulent, the ramp flow did not completely separate. The ramp has 
been redesigned to provide a zero pressure gradient at the anticipated separation point. Preliminary measurements with this new geometry show the flow to be separated for turbulent flow and separation control experiments will begin soon. Another experiment on transitory stall in conical diffusers is being planned. Tests should begin in December, 1987.

\subsubsection{Excitation of flow over a sphere and} dual cylinders. Effects of acoustic excitation have been experimentally studied on a sphere wake and on the flow around a pair of circular cylinders held normal to the free-stream. Upon excitation at appropriate frequencies, the reversed flow region behind a sphere was shortened, causing an increase in the form drag. 58 This contrasts the behavior of streamlined bodies where excitation has been observed to reduce drag. The flow between two circular cylinders "flip-flops" when they are spaced apart by less than one diameter. This flipping of the flow occurs because the wakes are asymmetric. With acoustic excitation the wakes can be made symmetric and the flipping can be prevented. This removes large unsteady loads on the cylinders.

\subsection{Grants and Contracts}

The following is a summary of research activities and some key results obtained so far under the current grant and contract program. Names of principal investigators are indicated.

3.2.1 Lockheed-Georgia Co. The research group led by Dr. K.K. Ahuja has performed important research on both the understanding and practical use of excitation in enhancing the mixing of jet flows. Hot jets, both subsonic and supersonic, have been the subject of extensive study, 13,59,60 and a large body of excitation data has been produced. If excitation is to be of practical use it is obvious that it must be effective for hot, high subsonic and supersonic jets. Disturbing trends were observed in the hot jet mixing data. 61 Although the mixing due to excitation of a low speed $(M=0.3)$ jet was increased at elevated temperature, the reverse trend was observed for high speed jets $(M=0.8)$. Above about $680 \mathrm{~K}$ no effect of excitation was observed. This anomaly between hot and cold jet mixing enhancement has recently been resolved and is the subject of a presentation in this conference. 62 Heating the jet decreased the Reynolds number causing the jet initial boundary layer state to become laminar or transitional. This apparently caused rapid mixing due to natural instabilities. As a result, additional single frequency excitation produced no further improvement. That the lack of "excitability" of the hot jet was due to an initial condition effect was demonstrated convincingly by tripping the initial boundary layer. The tripped hot jet behaved similar to the cold jet, and was found to be equally excitable. The initial condition effect may have also been responsible for an earlier observation by Jubelin, 63 who had reported a strong subharmonic in the noise spectra of an excited hot jet. A fully laminar initial boundary layer state, likely in his jet at the elevated temperature, could explain this observa tion.22,31 A subharmonic spike results from periodic pairing, which in turn requires a laminar boundary layer. 22 The initial condition effect on jet excitability, at lower Mach numbers for cold jets, has been studied in-house at NASA Lewis and will be reported in this conference. 29
A theoretical model has been developed at Lockheed by Tam and Morris 64 which calculates the velocity decay due to the mixing process in the jet. The theory was intended for use over a range of temperatures and velocities both with and without acoustic excitation. The predictions agreed with cold jet results but not with hot jet results. This is possibly due to the initial condition effect, as discussed above, which is not accounted for in the theory. (Dr. K.K. Ahuja).

3.2.2 Brown University. The theoretical study of multiple wave interactions in a spatially developing shear layer is being conducted. The theory uses the full set of conservation equations. The equations are averaged such that the mean flow, the coherent components and the fine grained turbulence are displayed. The coherent motion is split between odd and even modes thus providing a four element decomposition of the total flow. The mean flow is assumed to have a hyperbolic tangent type profile. The coherent structure components are assumed to have the transverse shape predicted by the eigenfuctions of the linear theory which recently has been shown to be quite adequate by Petersen and Samet. 65 The shear layer thickness, which characterizes the mean motion, and the coherent disturbance amplitudes must be jointly determined as a function of the distance from the origin of the shear layer. A two-component theory has been reported by Nikitopoulos and Liu. 66 This theory allows the analysis of the interaction of a fundamental disturbance frequency, the subharmonic, and the mean flow. The results show the phase between the two disturbances can be important in determining the development of the shear layer. The theory also shows the mixing "saturation" which occurs as the fundamental and then the subharmonic disturbances mature and decay. The theory is shown to yield results which closely duplicate the experimental results of Ho and Huang. 23 (Professor J.T.C. Liu).

3.2.3 University of Dayton. The mixing process in the shear layer of the axisymmetric jet is being studied using the full time-dependent Navier-Stokes equations which are numerically solved using MacCormack's explicit finite difference algorithm. Subscale turbulence models are not used in the calculations to date. External excitation can be applied through pressure or temperature perturbations at the upstream boundary of the jet pipe which simulates an upstream acoustic driver. Excitation has also been applied through pressure or temperature fluctuations on the pipe wall just inside the nozzle lip which simulates wall mounted acoustic drivers or heating strips. Scott 67 has compared his calculated large scale coherent structures to those observed experimentally by Lepicovsky et al.61 Experimental observations of the coherent structures including pairing activity as influenced by the excitation is quite well described by the computation. However, much higher excitation levels are required in the calculations $(160 \mathrm{~dB})$ than in the experiment $(144 \mathrm{~dB})$ for comparable structures. This difference is being investigated and might be as simple as a difference in measurement location. Some interesting points noted in the calculated results are: the coherent structures are qualitatively correctly predicted with the proper phase speed; pairing is observed as in the experiments; heat strip excitation just inside the jet lip is extremely effective; and the high speed hot jet is somewhat more excitable than 
the comparable cold jet. 68 Note the contrast of the last two points with the results of Refs. 43 and 20 . These are being investigated further. This numerical program should provide a useful tool for future enhanced mixing analysis, particularly when three-dimensional computation capability is added. (Professor J.N. Scott)

3.2.4 Michigan State University. Experiments are conducted on the behavior of a single stream. plane mixing layer. The mixing layer is of $80 \mathrm{~cm}$ span which can be studied in the $300 \mathrm{~cm}$ long test section. Controlled excitation can be applied by a full span piston located $2.84 \mathrm{~cm}$ upstream of the separation point. Reynolds number, based on the initial momentum thickness $\theta(0)$, as high as 5500 was obtainable. An interesting observation was the existence of a constant low frequency component, termed the ubiquitous freguency, throughout the entire entrainment domain. 69 This corresponded to a Strouhal number, $f_{\mu} \theta(0) / U_{0}=0.0018$. It is inferred that the formation of the first large scale coherent motion at $x / \theta(0)=400,70$ or at $260 \mathrm{~cm}$ in the MSU facility, is the source of the ubiquitous frequency. Excitation, with the conditions: $A=A_{0} \operatorname{Cos} \omega t$ where $A_{0}=3.6 \mathrm{~mm}$ or $A_{0} / \theta(0)=0.55$ and $\omega / 2 \pi=15 \mathrm{~Hz}=f_{F}$ or $f_{F} \theta(0) / U_{0}=0.0075 \mathrm{led}$ to: (1) a widened shear layer in terms of the mean velocity profile but (2) with no apparent increase in the mass flux through the test section and (3) a reduced ubiquitous frequency of $f_{u} \theta(0) / U_{0}=0.0014$. Further experiments are being conducted (Professor J.F. Foss).

3.2.5 University of Houston. The effect of excitation on the evolution of elliptic jets is studied experimentally. The effect has been found to be most pronounced at a Strouhal number, based on the equivalent diameter, of about 0.4 . Such excitation results in significantly enhanced mixing; 71 the jet cross-sectional area measured at about $x / D_{e}=5$ increases by as much as 190 percent compared to the unexcited jet case. A $4: 1$ elliptical jet has been found to be more susceptible to the excitation than a $2: 1$ jet. Excitation at $\mathrm{St}=0.85$ has been found to induce "jet column mode" of pairing, similarly as in an axisymmetric jet. In this case, however, the pairing process is more complicated and involves partial amalgamation of the nonaxisymmetric coherent structures. Far-field noise measurements have also been carried out in the elliptical jets. It is argued that pairing of the coherent structures should not be the principal noise source in jets. (Professor A.K.M.F. Hussain).

3.2.6 University of Arizona. The analysis of subharmonic resonance, in plane and axisymmetric mixing layers, is being carried out taking into account the effects of compressibility and temperature. Key results obtained so far show that increasing either the Mach number or the temperature reduces the growth rate of the subharmonic. "Weakly nonlinear" calculations for the stability of free shear layers is also being carried out. The calculation follows the temporal evolution of a wave packet. It has been shown that the wave packet must be imparted near the middle of the shear layer in order for it to receive maximum amplification. The magnitude of the "first Landau constant, "which provides an indication of the degree of the nonlinearity, has also been calculated systematically as a function of wave number and temperature. An interesting result is that the wavenumber components near the neutral point are found to suffer more nonlinearity than the ones receiving the maximum amplification. 72 (Professor T.F. Balsa).

Numerical analysis and experiments were carried out on the axisymmetric jet "preferred mode," which is defined as the frequency component of external forcing receiving maximum amplification by the jet. Contrary to earlier suggestions that the "preferred mode" is the result of global instability of the entire jet column or that it is an absolute instability, it is demonstrated that the preferred mode is actually a local, inflectional instability of the axisymmetric mixing layer. 65

The modal distribution of the coherent structures evolving near the nozzle of a circular jet were considered both analytically and experimentally. It has been shown that the cross-sectional shape of a jet can be distorted through the resonant interaction of two spinning modes with the same excitation frequency but with different circumferential mode numbers. Interactions between modes $\mathrm{m}=0$ and $\mathrm{m}=1$ and between modes $\mathrm{m}=0$ and $m=2$ have been considered, and it is demonstrated that, even on a time average basis, the circular cross-section of the jet undergoes distortion to oval or other shapes. Also investigated is the subharmonic resonance. The resonant interaction of a fundamental and a subharmonic of given mode numbers yielding a subharmonic of a different mode number, predicted analytically, is confirmed experimentalily. 15 (Professors R.A. Petersen and I. Wygnanski).

3.2.7 Ohio State University. Basic experimental research in a supersonic shear layer is being carried out. The study is to include mean and unsteady flow measurements in a supersonic shear layer emanating from a backward facing step. Two-component LOV measurements, Schlieren photography as well as a modified Schlieren measurement for preferred frequencies are to be performed. Some preliminary measurements have been carried out in an existing small scale supersonic wind tunnel, primarily addressing the validity of the measurement techniques. Construction of a larger supersonic wind tunnel, where the final experiments are to be done, is under way. (Professor M. Samimy).

\section{Research Plans at NASA Lewis}

Future research in the area of active control of flow should be divided into the categories of long term and short term efforts. The long term plan must include efforts which reveal the physics of the unsteady flow processes such that the role of the control signal is thoroughly understood. Basic work certainly seems to be needed. Chapman and Tobak 73 in contrasting the older statistical to the newer "structural" approach to turbulent flow research have stated, "in place of a theory without structure, the result to date has been structure without theory". Flow control, which largely stems from the concept that the coherent structure controls the flow and this structure can be controlled, directly suffers from the lack of understanding of the mechanisms involved. Research effort must concentrate on the longer term goal of providing models and theories for the unsteady, turbulent flow phencmena which we wish to control. One application which at present must be considered as long term is the manipulation and control of the extremely complex flows in the tip region of turbo- 
machinery. Future advances in efficiency and stall margin depend upon controlling this unsteady flow. Perhaps, tip treatment has already provided real istic control presently achievable in this flow. But one must strive for further improvement. Armed with a clearer understanding of the flows and with the inclusion of microcomputers and microsensors, the goal of obtaining significantly improved efficiency with active flow control, say within a decade, may be realistic.

Computer experiments involving the time dependent equations performing direct numerical simulation will be a great aid in advancing the knowledge of unsteady flows.74,75 Properly constructed computer solutions can provide detailed flow information which would be impossible to obtain any other way. These must be continuously validated by experimental measurements as the research progresses. The knowledge gained from the fundamental program in unsteady aerodynamics will be of great use in developing improved turbulence models upon which the progress of more conventional computational fiuid mechanics depends.

In the short term, many advances in flow control can be made which will help to maintain momentum in the research area. An important aspect of success in these practical applications will be the development of usable excitation devices. The short term advances can occur through the use of the best available current knowledge and some patient cutand-try techniques. We hope to achieve the first round of progress in practical control of inlet and diffuser flows, flows over airfoils, and significantly improved jet mixing in the near future.

\section{REFERENCES}

1. Schlichting, H., Boundary Layer Theory, 7th Edition, McGraw-Hi11, New York, 1979.

2. Schubauer, G.B. and Skramstad, H.K., "LaminarBoundary-Layer Oscillations and Transition on a Flat Plate," NACA Rept. 909, 1948.

3. Klebanoff, P.S., Tidstrom, K.D., and Sargent, L.M., "The Three-Dimensional Nature of Boundary Layer Instability, "Journal of Fluid Mechanics, Vol. 12, Pt. 1, Jan. 1962, pp. 1-34.

4. Craik, A.D.D., "Non-Linear Resonant Instability in Boundary Layers," Journal of Fluid Mechanics, Vol. 50, Pt. 2, Nov. 29, 1971, pp. 393-413.

5. Herbert, T., "Three-Dimensional Phenomena in the Transitional Flat Plate Boundary Layer," AIAA Paper 85-0489, Jan. 1985.

6. Kachanov, Y.S. and Levchenko, V.Y., "The Resonant Interaction of Disturbances at Laminar-Turbulent Transition in a Boundary Layer," Journal of Fluid Mechanics, Vol. 138, Jan. 1984, pp. 209-247.
7. Corke, T.C. and Mangano, R.A., "Transition of a Boundary Layer: Controlled FundamentalSubharmonic Interactions," Proceedings of the IUTAM Symposium on Turbulence Management and Relaminarization, Bangalore, India, Springer-Verlag, 1987.

8. Michalke, A., "On Spatially Growing Disturbances in an Inviscid Shear Layer, "Journal of Fluid Mechanics, Vol. 23, Pt. 3, Nov. 1965, pp. 521-544.

9. Freymuth, $P$. ., "On Transition in a Separated Laminated Boundary Layer," Journal of Fluid Mechanics, Vol. 25, Pt. 4, Aug. 1966, pp. 683-704.

10. Michalke, A., "Instability of a Compressible Circular Free Jet with Consideration of the Influence of the Jet Boundary Layer Thickness, " NASA TM-75190, 1977.

11. Crow, S.C. and Champagne, F.H., "Orderly Structure in Jet Turbulence," Journal of Fluid Mechanics, Vol. 48, Pt. 3, Aug. 16, 1971, pp. 547-591.

12. Bechert, D. and Pfizenmaier, E., "On Wavelike Perturbations in a Free Jet Travelling Faster than the Mean Flow in the Jet," Journal of Fluid Mechanics, Vol. 72, Pt. 2, Nov. 25, 1975, pp. 341-351.

13. Ahuja, K.K., Lepicovsky, J., Tam, C.K.W., Morris, P.J. and Burrin, R.H., "Tone-excited Jet -- Theory and Experiment," NASA CR-3538, 1982 .

14. Strange, P.J.R. and Crighton, D.G., "Spinning Modes on Axisymmetric Jets. Part I," Journal of Fluid Mechanics, Vol. 134, Sept. 1983, pp. 231-245.

15. Cohen, J. and Wygnanski, I., "The Evolution of Instabilities in the Axisymmetric Jet," Journal of Fluid Mechanics, Vol. 176, Mar. 1987, pp. $191-235$.

16. Hussain, A.K.M.F. and Thompson, C.A., "Controlled Symmetric Perturbation of the Plane Jet: An Experimental Study in the Initial Region," Journal of Fluid Mechanics, Vol. 100, Pt. 2, Sept. 25, 1980, pp. 397-43i.

17. Coles, D. and Barker, S.J., Turbulent Mixing in Nonreactive and Reactive Flows, S.N.B. Murthy, ed., Plenum Press, New York, 1975 , p. 285 .

18. Gutmark, E. and Ho, C.-M., "Near-Field Pressure Fluctuations of an Elliptic Jet," AIAA Journal, Vol. 23, No. 3, Mar. 1985, pp. 354-358.

19. Husain, H.S. and Hussain, A.K.M.F., "Controlled Excitation of Elliptic Jets, "Physics of Fluids, Vol. 26, No. 10, Oct. 1983, pp. $2763-2766$.

20. Ahuja, K.K., Lepicovsky, J. and Brown, W.H., "Some Unresolved Questions on Hot-Jet Mixing Control Through Artificial Excitation, "AIAA Paper 86-1956, July 1986. 
21. Brown, G.L. and Roshko, A., "On Density Effects and Large Structures in Turbulent Mixing Layers, "Journal of Fluid Mechanics, Vol. 64, Pt. 4, July 24, 1974, pp. 775-816.

22. Hussain, A.K.M.F. and Zaman, K.B.M.Q., "Vortex Pairing in a Circular Jet Under Controlled Excitation. Part 2. Coherent Structure Dynamics," Journal of Fluid Mechanics, Vol. 101, Pt. 3, Dec. 11, 1980, pp. 493-544.

23. Ho, C. M. and Huang, L.-S., "Subharmonics and Vortex Merging in Mixing Layers," Journal of Fluid Mechanics, Vol. 119, June 1982, pp. 443-473.

24. Oster, D., Wygnanski, I., Dziomba, B. and Fiedler, $H$. , "On the Effect of Initial Conditions on the Two-dimensional Turbulent Mixing Layer," Structure and Mechanisms of Turbulence, I, H. Fiedler, ed., Springer-Verlag, New York, 1977 , pp. $48-64$.

25. Mankbadi, R. and Liu, J.T.C., "A Study of the Interactions Between Large-Scale Coherent Structures and a Fine-grained Turbulence in a Round Jet," Philosophical Transactions of the Royal Society of London, Series A, Vol. 298, No. 1443, Jan. 21, 1981, pp. 541-602.

26. Bushnel1, D.M., "Turbulent Drag Reduction for External Flows," AIAA Paper 83-0227, Jan. 1983.

27. Reshotko, E., "Control of Boundary Layer Transition," AIAA Paper 85-0562, Mar. 1985.

28. Stone, J.R. and McKinzie, J.D., Jr., "Acoustic Excitation - - a Promising New Means of Controlling Shear Layers," NASA TM-83772, 1984.

29. Raman, G., Zaman, K.B.M.Q. and Rice, E.J., "Initial Turbulence Effects on Jet Excitability," AIAA Paper 87-2725, Oct. 1987.

30. Zaman, K.B.M.Q. and Hussain, A.K.M.F., "Turbulence Suppression in Free Shear Flows by Controlled Excitation," Journal of Fluid Mechanics, Vol. 103, Feb. 1981 pp. 133-160.

31. Zaman, K.B.M.Q., "Far-Field Noise of a Subsonic Jet Under Controlled Excitation," Journal of Fluid Mechanics, Vol. 152, Mar. 1985, pp. 83-111.

32. Liepmann, H.W., Brown, G.L., and Nosenchuck, D.M., "Control of Laminar-Instability Waves Using a New Technique," Journal of Fluid Mechanics, Vol. 118, May 1982, pp. 187-200.

33. Thomas, A.S.W., "The Control of BoundaryLayer Transition Using a Wave-Superposition Principle," Journal of Fluid Mechanics, Vol. 137, Dec. 1983, pp. 233-250.

34. Maestrello, L. and Ting, L., "Analysis of Active Control by Surface Heating," AIAA Journal. Vol. 23, No. 7, July, 1985, pp. 1038-1045.

35. Mangalam, S.M., Bar-Sever, A., Zaman, K.B.M.Q., and Harvey W.D., "Transition and Separation Control on a Low-Reynolds Number Airfoll," Proceedings International Conference on Aerodynamics at Low Reynolds Numbers, London, 1986.
36. Ahuja, K.K. and Burrin, R.H., "Control of Flow Separation by Sound," AIAA Paper 84-2298, Oct. 1984.

37. Zaman, K.B.M.Q., Bar Sever, A., and Mangalam, S.M., "Effect of Acoustic Excitation on the Flow Over a Low-Re Airfoil " Journal of Fluid Mechanics, Vol. 182, Sept. 1987, pp. 127-148.

38. Epstein, A.H., Ffowes Williams, J.E. and Greitzer, E.M., "Active Suppression of Compressor Instabilities," AIAA Paper 86-1914, July 1986.

39. McCroskey, W.J., "Recent Developments in Dynamic Sta 11," Unsteady Aerodynamics, Vol. 1, R.B. Kinney, ed., Univ. of Arizona, Tuscon, $A \dot{Z}$, 1975, pp. 1-34.

40. McCroskey, W.J., Carr, L.W. and MCAl ister, K.W., "Dynamic Stall Experiments on Oscillating Airfoils," AIAA Paper 75-125, Jan. 1975.

41. Viets, H., Ball, M., and Bougine, D., "Performance of Forced Unsteady Diffusers," AIAA Paper 81-0154, Jan. 1981.

42. Simpson, R.L., "Two-Dimensional Turbulent Separated Flow," AGARD AG-287-VOL-1, AGARD, Neuilly Sur Seine, France, 1986.

43. Strykowski, P.J. and Sreenivasan, K.R., "The Control of Transitional Flows," AIAA Paper 85-0559, Mar. 1985.

44. Leehey, P. and Shapiro, P., "Leading Edge Effect in Laminar Boundary Layer Excitation by Sound," Laminar Turbulent Transition, R. Eppler and H. Fasel, eds., Springer-Verlag, New York, 1979, pp. 321-331.

45. Goldstein, M.E., "The Evolution of TollmienSchlichting Waves Near a Leading Edge," Journal of Fluid Mechanics, Vol. 127, Feb. 1983, pp. 59-81.

46. Kibens, V., "Discrete Noise Spectrum Generated by an Acoustically Excited Jet," AIAA Paper 79-0592, Mar. 1979.

47. Perry, A.E., Lim, T.T. and Teh, E.W., "A Visual Study of Turbulent Spots," Journal of Fluid Mechanics, Vol. 104, Mar. 1981, pp. 387-405.

48. Sokolov, M., Kleis, S.J., and Hussain, A.K.M.F., "Coherent Structures Induced by Two Simultaneous Sparks in an Axisymmetric Jet," AIAA Journal, Vol. 19, No. 8, Aug. 1981, pp. $1000-1008$.

49. Taghavi, R., Rice, E.J., and Farokhi, S., "Controlled Excitation of a Cold Turbulent Swirling Free Jet," Journal of Vibration Acoustics, Stress and Reliability in Design, (to appear), 1987.

50. Rossiter, J.E., "Wind-tunnel Experiments on the Flow Over Rectangular Cavities at Subsonic and Transonic Speeds," RAE TR-64037, ARC R\&M-3438, Oct. 1964. 
51. Rockwe 11, D. and Naudascher, E., "SelfSustaining Oscillations of Flow Past Cavities," Journal of Fluids Engineering, Vol. 100, No. 2, June 1978, pp. 152-165.

52. Komerath, N.M., Ahuja, K.K., and Chambers, F.W., "Prediction and Measurement of Flows Over Cavities -- a Survey," AIAA Paper 87-0166, Jan. 1987.

53. Viets, H., "Flip-Flop Jet Nozzle," AIAA Journal, Vol. 13, No. 10, Oct. 1975, pp. 1375-1379.

54. Hasan, M.A.Z. and Hussain, A.K.M.F., "The Self-Excited Axisymmetric Jet." Journal of Fluid Mechanics, Vol. 115, Feb. 1982, pp. 59-89.

55. Michalke, A., "Survey on Jet Instability Theory, "Progress in Aerospace Sciences, Vol. 21, No. 3, 1984, pp. 159-199.

56. Miles, J.H., "Spatially Growing Disturbances in a Two-Stream, Coplanar Jet," AIAA Paper 86-0041, Jan. 1986. (NASA TM-88949).

57. Miles, J.H., "Spatially Growing Disturbances in a High Velocity Ratio Two-Stream, Coplanar Jet," AIAA Paper 87-0056, Jan. 1987 (NASA TM-88922).

58. Kim, H.J. and Durbin, P.A., "Response of a Sphere Wake to Acoustic Excitation," Journal of Fluid Mechanics (submitted), 1987.

59. Ahuja, K.K., "Basic Experimental Study of the Coupling Between Flow Instabilities and Incident Sound," NASA CR-3789, 1984.

60. Lepicovsky, J., Ahuja, K.K., Brown, W.H. and Burrin, R.H., "Coherent Large-Scale Structures in High Reynolds Number Supersonic Jets," NASA CR-3952, 1985.

61. Lepicovsky, J., Ahuja, K.K., Brown, W.H., and Morris, P.J., "Acoustic Control of Free Jet Mixing," Journal of Propulsion and Power Vol. 2, No. 4, July-Aug. 1986, pp. 323-330.

62. Lepicovsky, J. and Brown, W.H., "Effects of Nozzle Exit Boundary-Layer Conditions on Excitability of Heated Free Jets," AIAA Paper 87-2723, Oct. 1987

63. Jubelin, B., "New Experimental Studies on Jet Noise Amplification," AIAA Paper 80-0961, June 1980.

64. Tam, C.K.W. and Morris, P.J., "Tone Excited Jets, Part V; A Theoretical Model and Comparison With Experiment." Journal of Sound and Vibration, Vol 102, No. 1, Sept. 8, 1985 . pp. 119-151.
65. Petersen, R.A. and Samet, M.M., "On the Preferred Mode of Jet Instability," Journal of Fluid Mechanics (submitted), 1987.

66. Nikitopoulos, D.E. and Liu, J.T.C., "Nonlinear Binary-Mode Interactions in a Developing Mixing Layer", Journal of Fluid Mechanics, Vol. 179 June 1987, pp. 345-370.

67. Scott, J.N., "Numerical Simulation of Self Sustained and Forced Oscillations in Jet Shear Layers," ASME Fluids Engineering Spring Conference, Forum on Unsteady Flow Separation, Cincinnati, Ohio, June 15-17, 1987.

68. Scott, J.N. and Hoo, E.A., "A Numerica! Investigation of the Influence of Heating on the Excitation of $\mathrm{High}$ and Low Mach Number Jet Flows," to be presented at the ASME 1987 winter Annual Meeting, Boston, MA, Dec. 13-18, 1987.

69. Ali S.K., Klewicki, C.L., Disimile, P.J. Lawson, I., and Foss, J.F.. "Entrainment Region Phenomena for a Large Plane Shear Layer," 5 th Symposium on Turbulent Shear Flows. Pennsylvania State University, 1985. pp. 3.7-3.12.

70. Hussain, A.K.M.F. \& Zaman, K.B.M.Q., "An Experimental Study of Organized Motions in the Turbulent Plane Mixing Layer," Journal of Fluid Mechanics, Vol. 159, Oct. 1985, pp. 85-104.

71. Hussain, A.K.M.F. and Husain, H.S., "Passive and Active Control of Jet Turbulence," IUTAM Symposium on Turbulence Management and Relaminarization, Bangalore, India, Springer-Verlag, 1987

72. Balsa, T.F., "On the Spatial Instability of Piecewise Linear Free Shear Layers," Journal of Fluid Mechanics, Vol. 174, Jan. 1987, pp. 553-563.

73. Chapman, G.T. and Tobak, M., "Observations, Theoretical Ideas, and Modeling of Turbulent Flows - Past, Present, and Future," NASA TM-86679, 1985

74. Claus, R.W., "Direct Numerical Simulations of a Temporally Evolving Mixing Layer Subject to Forcing," NASA TM-88896, 1986.

75. Metcalfe, R.W., Orszag, S.A., Brachet, M.E., Menon, S., and Riley, J.J., "Secondary Instability of a Temporally Growing Mixing Layer," Journal of Fluid Mechanics. (submitted), 1987. 
TABLE 1. - CHART SHOWING VARIOUS USES OF ARTIFICAL EXCITATION

Use of Artifical Excitation

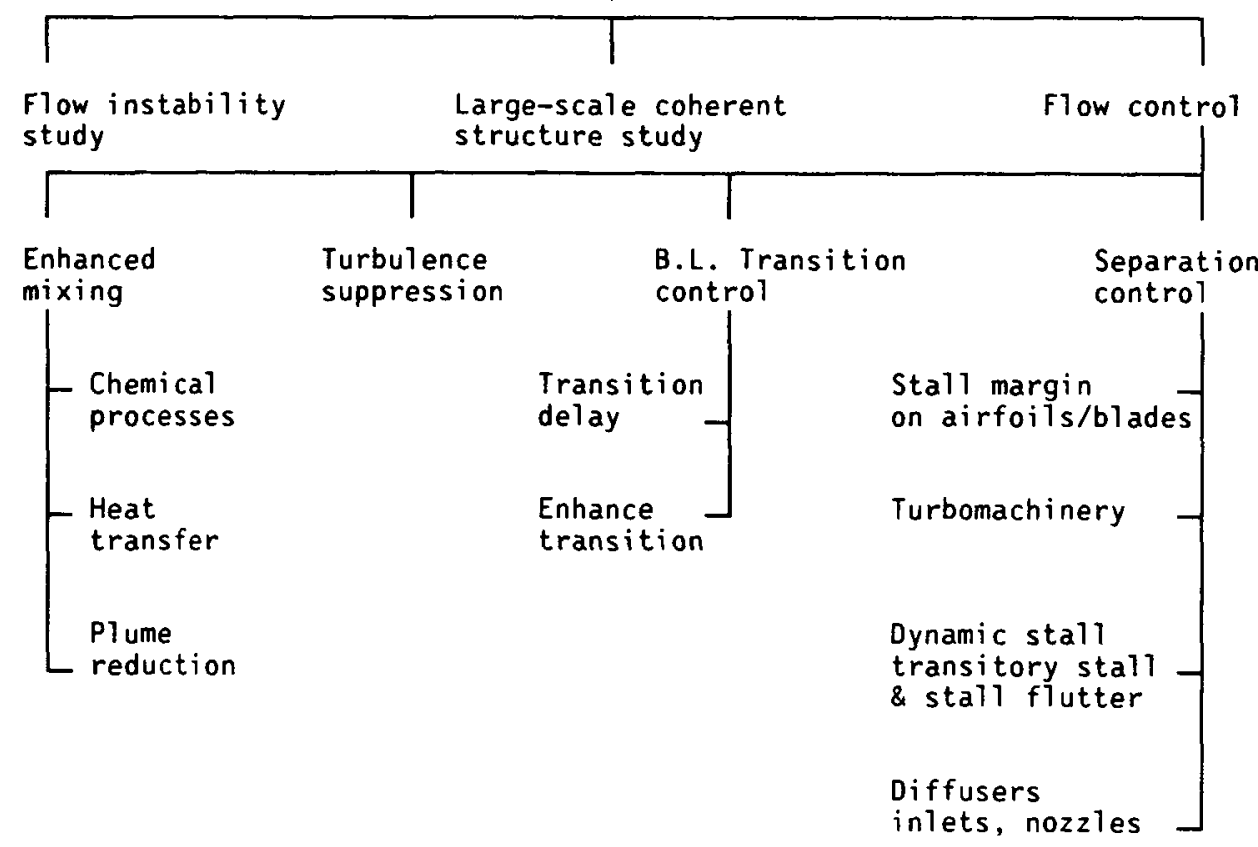




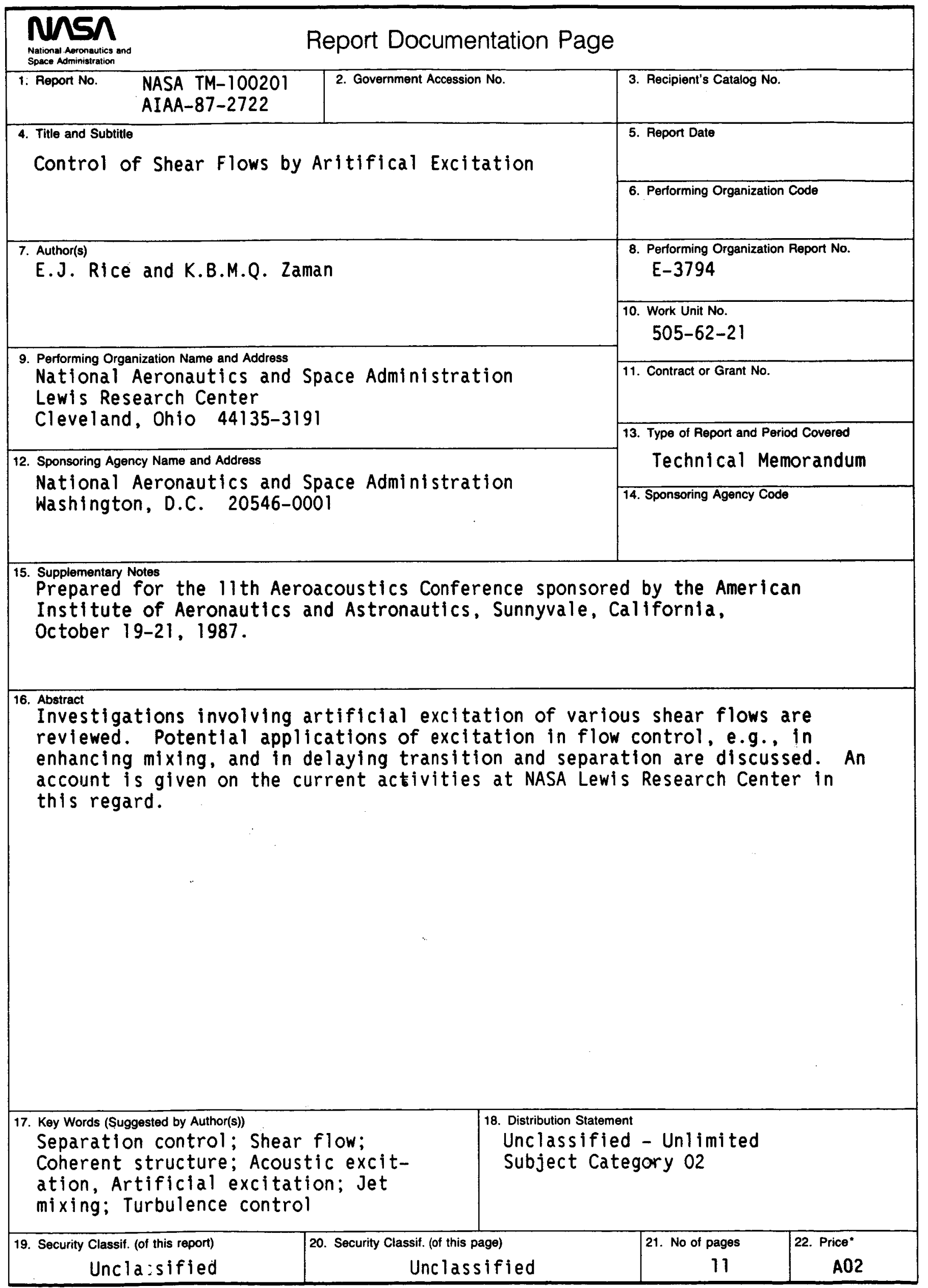

NASA FOAM 1626 OCT $86 \quad$ "For sale by the National Technical Information Service, Springfield, Virginia 22161 